\title{
Measurements of $\Delta G$
}

\author{
Gerhard Mallot
}

CERN, 1211 Geneva, Switzerland

\begin{abstract}
Our present information on the gluon polarisation $\Delta g / g$ is reviewed. The data from fixedtarget lepton-nucleon experiments are in context with the recent data from the RHIC polarised pp collider. The main tools to study $\Delta g / g$ in lepton-nucleon scattering are scaling violations of the $g_{1}$ structure functions and longitudinal spin asymmetries in hadron production. Results from high- $p_{\mathrm{T}}$ hadron pairs, inclusive hadrons as well as open-charm production are discussed. At RHIC the most precise data presently came from inclusive $\pi^{0}$ and jet production. All data indicate that the gluon polarisation is small compared to earlier expectations, but still can make a major contribution to the nucleon spin.
\end{abstract}

Keywords: Gluon polarisation, spin asymmetry, parton distribution functions

PACS: $14.20 . \mathrm{Dh}$ 14.70.Dj 13.60.Hb 14.65.Bt 14.65.Dwd

\section{INTRODUCTION}

Following the discovery [1] in 1987 by the European Muon Collaboration that the first moment $\Gamma_{1}$ of spin-dependent structure function $g_{1}$ of the proton is much smaller than expected from the Ellis-Jaffe sum rule, the spin structure of the nucleon became a focus in theoretical and experimental research. By now it is experimentally well established that indeed the matrix element $a_{0}$ of the flavour-singlet axial-vector current is small and only in the order of 0.2-0.3. If the quark spins were responsible for the nucleon spin a value around 0.6 is expected. In the $\overline{\mathrm{MS}}$ renormalisation scheme the sum of the quark spins $\Delta \Sigma=\Delta u+\Delta d+\Delta s$ is given by $a_{0}$, while in the so-called Adler-Bardeen and JET schemes $a_{0}$ receives an additional contribution from the gluon polarisation $\Delta G=\int \Delta g\left(x, Q^{2}\right) \mathrm{d} x$ of $-\frac{n_{f} \alpha_{s}}{2 \pi} \Delta G$. This led to the conjecture that a large positive $\Delta G$ could explain the smallness of $a_{0}$ and at the same time reestablish the expected contribution of 0.6 from the quark spins to the nucleon spin. However, in order to respect the spin sum rule $\frac{1}{2}=\frac{1}{2} \Delta \Sigma+\Delta G+L_{z}$ a large gluon polarisation requires partial cancellation by orbital angular momentum $L_{z}$. The key to solving the nucleon spin puzzle is thus a measurement of gluon polarisation. Now, two decades after the original discovery, we hold in hands first data suggesting that the large $\Delta G$ scenario is not realised in nature.

\section{SCALING VIOLATIONS}

The $Q^{2}$ evolution of structure functions is governed by the DGLAP equations. Thus the polarised gluon distribution function $\Delta g\left(x, Q^{2}\right)$ can in principle be obtained from a QCD fit to the world data on the spin-dependent structure function $g_{1}\left(x, Q^{2}\right)$. In the unpolarised case, HERA and fixed-target data combined offer a huge range in $x$ and 


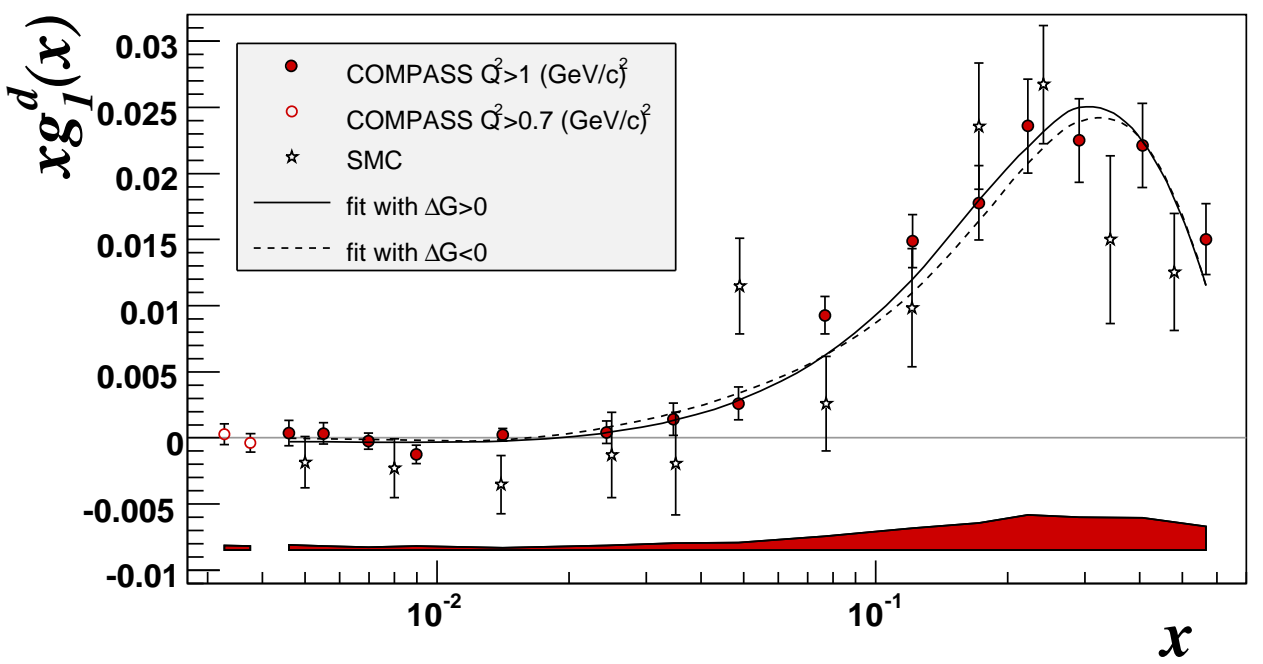

FIGURE 1. New $x g_{1}\left(x, Q^{2}\right)$ deuteron data from COMPASS as function of $x$ together with the NLO QCD fit results [3].

$Q^{2}$. Due to the lack of a polarised ep collider the situation is completely different in the polarised case. Here the QCD fits rely largely on the rather small difference in $Q^{2}$ between the SLAC/HERMES data on one side and the SMC/COMPASS data on the other side, spanning c.m. energies from $8 \mathrm{GeV}$ to $20 \mathrm{GeV}$. The status of QCD fits was discussed in detail this Symposium [2]. In the small $x$ region the new deuteron $g_{1}$ data from COMPASS [3] are about six times more precise than the SMC data. CoMPASS performed next-to-leading order (NLO) fits to the $g_{1}$ world data including the new deuteron data. Two about equally good solutions for $\Delta g\left(x, Q^{2}\right)$ were found, one with a positive and one with a negative first moment $\Delta G$ (Figs. 1 and 2). The absolute value is in the order of $|\Delta G| \simeq 0.2-0.3$ for $Q_{0}^{2}=3 \mathrm{GeV}^{2}$ and the uncertainty from the fit is in the order of 0.1 . Contributions to the error arising from uncertainties in the factorisation and renormalisation scales as well as the influence of the particular parametrisation chosen for the distribution functions, were not considered.

Recent fits by the Asymmetry Analysis Collaboration (AAC) [4] to the world DIS data (including only a part of the COMPASS data) also find solutions with negative and positive first moments $\Delta G$ at $Q_{0}^{2}=1 \mathrm{GeV}^{2}$. In a first step toward a global analysis of data sensitive to $\Delta g$, they included the $\pi^{0}$ helicity asymmetries [5] from the PHENIX 2005 run (see below) in the fit (Fig. 2). A very significant reduction of the uncertainty of $\Delta G$ from 1.08 to 0.32 was observed, at least for the $\Delta G>0$ solution. However, the sign ambiguity remains. The contribution to $\Delta G$ from the region $x>0.1$ is 0.3 for both solutions. The negative first moment for the $\Delta G<0$ solution stems entirely from the $x<0.1$ region. In contrast to the COMPASS fit, the AAC fit yields a gluon distribution with a node around $x=0.16$.

It should be pointed out that the two groups apply different error estimations and thus the error bands shown have different meanings. While the COMPASS error band corresponds to the change of $\chi^{2}$ by unity, the AAC bands corresponds to a change of $\chi^{2}$ 

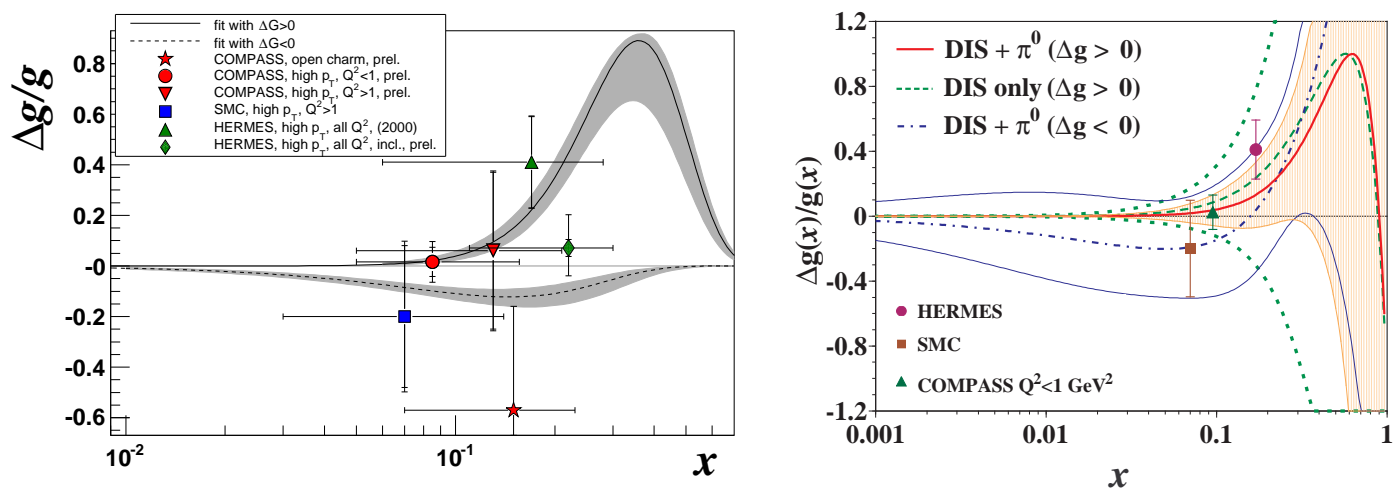

FIGURE 2. Gluon polarisation $\Delta g / g$ as function of $x$ at $Q^{2}=Q_{0}^{2}$ obtained by NLO QCD fits (bands) and from LO analysis of hadron helicity asymmetries (symbols). Left: from COMPASS QCD fits [3] including the new COMPASS deuteron data. $\left(Q_{0}^{2}=3 \mathrm{GeV}^{2}\right)$; Right: from AAC QCD fits [4] involving the $\pi^{0}$ helicity asymmetries from PHENIX [5] $\left(Q_{0}^{2}=1 \mathrm{GeV}^{2}\right)$.

by 12.65 for their eleven free parameters, what corresponds to $63 \%$ probabilty to find all parameters simultaneously within one standard deviation rather than an individual parameter. For details see the documentation of the MINUIT program [6].

\section{HIGH- $p_{\top}$ HADRONS IN LEPTO-PRODUCTION}

To probe the gluon polarisation helicity asymmetries were studied for the inclusive production of hadrons, the production of hadron pairs and of charmed hadrons $(D$ mesons). For inclusive hadrons [7] and open-charm [8] NLO calculations exist, while for hadron pairs NLO is in progress and LO is available [9]. However, up to now the analyses were performed in leading order. Sensitive to the gluon polarisation are the gluon-photon fusion (PGF) and the resolved photon parton-level processes. For the latter the polarised PDFs of the resolved photon need to be known. The QED part can be calculated and the remaining part is bounded by the unpolarised PDFs. For hadron-pair production with high transverse momentum $p_{\mathrm{T}}$ and $Q^{2}>1 \mathrm{GeV}^{2}$ as well as for opencharm production kinematic regions can be chosen where the PGF process dominates. The longitudinal double-spin asymmetry $A_{\|}$is then in leading order linear in the gluon polarisation

$$
A_{\|}=R_{\mathrm{pgf}} a_{\mathrm{pgf}} \frac{\Delta g}{g}+A_{\mathrm{bgd}}
$$

where $R_{\mathrm{pgf}}$ is the fraction of PGF events and $a_{\mathrm{pgf}}$ is analysing power of the PGF subprocess. The latter can be calculated for a given kinematics, but for a particular measurement both, $R_{\mathrm{pgf}}$ and the average $a_{\mathrm{pgf}}$, have to be estimated by Monte Carlo (MC) simulations. This introduces a model dependence in the determination of $\Delta g / g$. A possible background asymmetry $A_{\text {bgd }}$ arising e.g. from QCD-Compton and direct processes needs also to be estimated. The resulting value of $\Delta g / g$ represents an average of $\Delta g / g(x)$ over the probed $x_{g}$ range, which needs to be determined from the MC simulation. The presently 
TABLE 1. Leading order measurements of $\Delta g / g$

\begin{tabular}{llccccrr}
\hline Experiment & \multicolumn{1}{c}{ Method } & $\Delta g / g$ & $\begin{array}{r}\text { Stat. } \\
\text { Error }\end{array}$ & $\begin{array}{r}\text { Sys. } \\
\text { Error }\end{array}$ & $\begin{array}{r}\left\langle\mu^{2}\right\rangle \\
\mathbf{G e V}^{2}\end{array}$ & $\langle x\rangle$ & Published \\
\hline COMPASS & hadron pairs $\left(Q^{2}<1\right)$ & 0.016 & 0.058 & 0.055 & $\sim 3$ & 0.085 & prel., [10] \\
COMPASS & hadron pairs $\left(Q^{2}>1\right)$ & 0.06 & 0.31 & 0.06 & & 0.13 & prel. \\
COMPASS & open charm & -0.57 & 0.41 & & 13 & 0.15 & prel. \\
HERMES & hadron pairs & 0.41 & 0.18 & 0.03 & $\sim 2$ & 0.17 & {$[11]$} \\
HERMES & incl. hadrons & 0.071 & 0.034 & -0.105 & 1.35 & 0.22 & {$[12]$} \\
SMC & hadron pairs $\left(Q^{2}>1\right)$ & -0.20 & 0.28 & 0.10 & & 0.07 & {$[13]$} \\
\hline
\end{tabular}
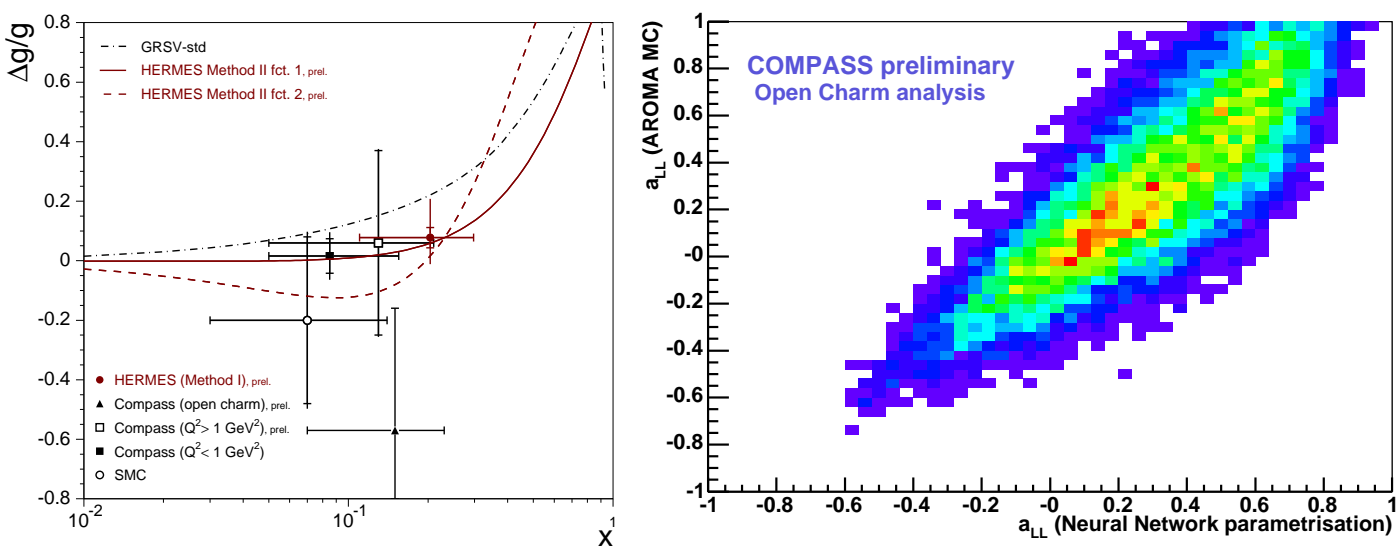

FIGURE 3. Left: Hermes $\Delta g / g$ resulting from fits to data in $p_{\mathrm{T}}$ bins (Method II). Also shown is a point extracted assuming a constant $\Delta g / g$ (Method I). Right: COMPASS open charm, correlation between the true $a_{\mathrm{PGF}}$ from MC and $a_{\mathrm{PGF}}$ obtained using a neural network trained on event kinematics.

available determinations of $\Delta g / g$ from hadron lepto-production are summarised in Table 1 and shown in Fig. 2 .

COMPASS performed separate analyses for hadron pairs produced at $Q^{2} \geq 1 \mathrm{GeV}^{2}$ and $Q^{2}<1 \mathrm{GeV}^{2}$. For both samples transverse momentum cuts of $p_{\mathrm{T}}$ with $p_{\mathrm{T}, \mathrm{h} 1}^{2}+p_{\mathrm{T}, \mathrm{h} 2}^{2} \geq$ $2.5 \mathrm{GeV}^{2}$ were applied. The inclusion of the 2004 deuteron data in the low $Q^{2}$ analysis [10] yielded an about 1.5 times more precise preliminary result. The gluon polarisation from the high and low $Q^{2}$ analyses are compatible with zero and probe the region around $x_{g} \simeq 0.1$.

HERMES presented new analyses of $\Delta g / g$ [12] including the deuteron data. Their most precise result comes from inclusive hadron asymmetries. A polarised gluon PDF is fitted to $\Delta g / g$ in four $p_{\top}$ bins in the range $1.05 \mathrm{GeV}<p_{\top}<2.5 \mathrm{GeV}$ ('Method II'). Figure 3 (left) shows two of these fits using different functional forms for $\Delta g / g$. Also shown is a new HERMES point for $\Delta g / g$ extracted from the same data, but assuming that $\Delta g / g$ is constant over the probed $x_{g}$ range (Method I). The $x_{g}$ range indicated in Fig. 2 (left) comes from this point rather than from 'Method II'. The resulting gluon polarisation is again very small and most sensitive to the region $x_{g} \simeq 0.2$. The larger 1999 HERMES result [11] had been obtained from hadron pairs and the data are included in the new analysis. 

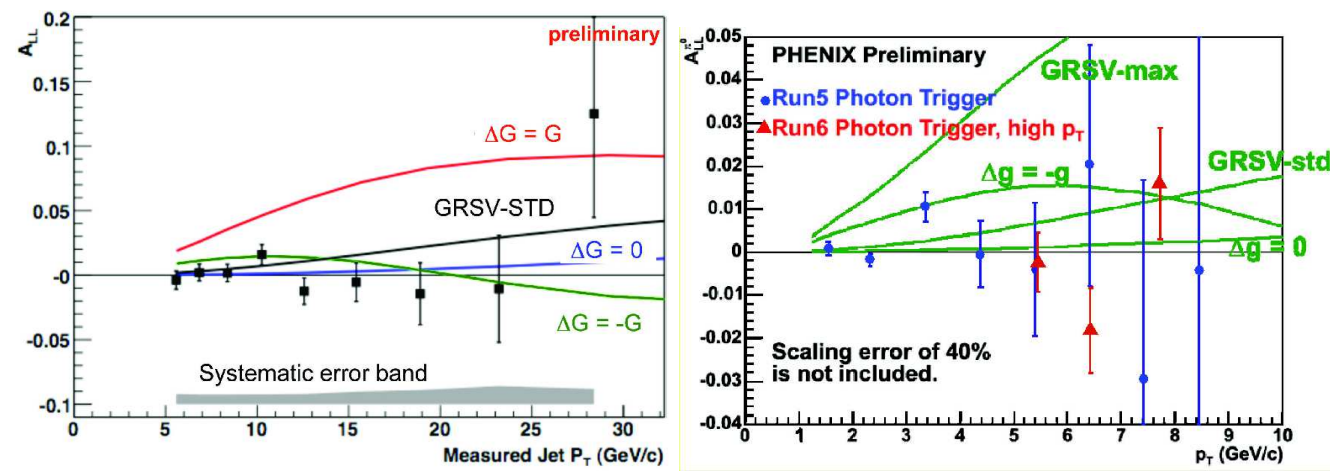

FIGURE 4. $A_{\mathrm{LL}}$ as function of $p_{\mathrm{T}}$. Left: $A_{\mathrm{LL}}$ for inclusive jet production at STAR [14]. Right: $A_{\mathrm{LL}}$ for inclusive $\pi^{0}$ production at PHENIX [5].

Open charm production is considered the most model-independent tool to study the gluon polarisation. Due to the small initial cross-sections and the small branching ratio in the decay $D^{\circ} \rightarrow K \pi$, the measurement of an asymmetry in $D$ meson production is a real challenge. COMPASS has determined $\Delta g / g$ obtained from this asymmetry. A neural network was used to estimate $a_{\mathrm{PGF}}$ from the kinematics on an event-by-event basis. The correlation of the network response and the true $a_{\mathrm{PGF}}$ is shown is Fig. 3 (right). The preliminary result is smaller than - but compatible with - zero (Table 1, Fig. 2]left).

\section{RHIC DATA}

The polarised $p p$ collider RHIC offers many channels to study the gluon polarisation. At present luminosities and a c.m. energy of $\sqrt{s}=200 \mathrm{GeV}$ the most promising channels are the inclusive $\pi^{0}$ [5] and jet [14] longitudinal double-spin asymmetries $A_{\mathrm{LL}}$ measured by PHENIX and STAR. The present status of these measurements is shown Fig. 4 . Also shown are NLO calculations [15, 16] using the GRSV set of PDFs [17] for four different assumptions for the gluon polarisation, namely the best fit to the world data (GRSV-std) and $\Delta g=-g, 0, g$ at $Q_{0}^{2}=0.3 \mathrm{GeV}^{2}$. As first observed by COMPASS [10] the data rule out the $\Delta g=g$ scenario, while the other scenarios are still possible. The dependence of the asymmetry $A_{\mathrm{LL}}$ on $\Delta g / g$ contains a quadratic term which makes it at present impossible to determine the sign of $\Delta g / g$ from RHIC data. This will improve with the $p_{\mathrm{T}}$ range available in the future at $\sqrt{s}=500 \mathrm{GeV}$. Very encouraging are the prospects for the data taken in 2006. The average beam polarisation $P_{\mathrm{B}}$ improved from $46 \%$ in 2005 to $62 \%$ and the also luminosity $L$ increased considerably. The figure of merit is proportional to $L P_{\mathrm{B}}^{4}$ and the 'Run 6 high $p_{\mathrm{T}}$ ' PHENIX data allow us a first glimpse on the precision of the new data (Fig. 4 right). Projections by STAR [14] for the precision of the 2006 jet data are very promising and should allow to distinguish between the GRSV-std and the GRSV-min scenarios. 


\section{OUTLOOK}

New data from COMPASS and RHIC will further clarify the composition of the nucleon spin. The restoration of a $60 \%$ contribution of the quark spins via the axial anomaly is basically ruled out. The new challenge is to precisely determine the fraction of the nucleon spin carried by gluon spins rather than distinguishing between the various scenarios of the 'spin puzzle'. This must include a precise pinning down of the $x$ dependence of $\Delta \mathrm{g} / \mathrm{g}$. For this purpose a global analysis of all relevant data is indispensable. A particular effort needs to be made to include the lepton data in a consistent way in the NLO analyses.

The second goal must be a measurement of the angular momentum contribution to the nucleon spin. Presently it looks a little bit like all candidates contribute about equally to the nucleon spin, a scenario which is particularly hard to prove experimentally.

\section{ACKNOWLEDGMENTS}

I thank the organisers for a wonderful and inspiring Symposium, P. Liebig and E. Aschenauer for interesting discussions on the new HERMES result, N. Saito for details on the AAC fit, and my colleagues from the COMPASs Collaboration for the support in selecting and interpreting the material.

\section{REFERENCES}

1. EMC, J. Ashman et al. Nucl. Phys. B 328, 1 (1989).

2. J. Blümlein, session $2 \mathrm{~A}$, these proceedings.

3. COMPASS, I. Savin, session 2A, these proceedings;

V. Yu. Aleksakhin et al., subm. to Phys. Lett., arXiv:hep-ex/0609038

4. AAC, M. Hirai, session 2A, these proceedings;

M. Hirai, S. Kumano and N. Saito, Phys. Rev. D 74, 014015 (2006), [arXiv:hep-ph/0603213].

5. PHENIX, K. Boyle, session 2A, these proceedings.

6. F. James and M. Roos, Comput. Phys. Commun. 10, 343 (1975); http://wwwasdoc.web.cern.ch/wwwasdoc/minuit/minuit.ps.gz

7. B. Jäger, M. Stratmann and W. Vogelsang, Eur. Phys. J. C 44, 533 (2005) [arXiv:hep-ph/0505157].

8. I. Bojak and M. Stratmann, Phys. Lett. B 433, 411 (1998) [arXiv:hep-ph/9804353]; Nucl. Phys. B 540, 345 (1999) [arXiv:hep-ph/9807405].

9. C. Hendlmeier, M. Stratmann and A. Schäfer, Eur. Phys. J. C 48, 135 (2006) [arXiv:hep-ph/0606096].

10. E. S. Ageev et al. [Compass Collaboration], Phys. Lett. B 633, 25 (2006) [arXiv:hep-ex/0511028].

11. Hermes, A. Airapetian et al., Phys. Rev. Lett. 84, 2584 (2000) [arXiv:hep-ex/9907020].

12. Hermes, P. Liebig, session $2 \mathrm{~A}$, these proceedings.

13. SMC, B. Adeva et al., Phys. Rev. D 70, 012002 (2004) [arXiv:hep-ex/0402010].

14. STAR, D. Relyea, session $2 \mathrm{~A}$, these proceedings.

15. B. Jäger, M. Stratmann and W. Vogelsang, Phys. Rev. D 70, 034010 (2004) [arXiv:hep-ph/0404057].

16. B. Jäger, A. Schafer, M. Stratmann and W. Vogelsang, Phys. Rev. D 67, 054005 (2003) [arXiv:hep-ph/0211007].

17. M. Glück, E. Reya, M. Stratmann and W. Vogelsang, Phys. Rev. D 63, 094005 (2001) [arXiv:hep-ph/0011215]. 